\title{
Selective entorhinal and nonselective cortical- hippocampal region lesions, but not selective hippocampal lesions, disrupt learned irrelevance in rabbit eyeblink conditioning
}

\author{
M. TODD ALLEN, LORI CHELIUS, and MARK A. GLUCK \\ Rutgers University, Newark, New Jersey
}

\begin{abstract}
Prior experiments, as well as computational models, have implicated the hippocampal region in mediating the influence of nonreinforced stimulus preexposure on subsequent learning. Learned irrelevance (LIRR) is a preexposure task in which uncorrelated preexposures to the conditioned stimulus (CS) and the unconditioned stimulus (US) produce a retardation of subsequent CS-US conditioning. In the work presented here, we report the results of tests of LIRR in eyeblink conditioning in rabbits with sham lesions, nonselective cortical-hippocampal region lesions, selective hippocampal lesions, and selective entorhinal lesions. Sham-lesioned rabbits that had been preexposed to the CS and the US exhibited slower acquisition of conditioned responses, as compared with context-preexposed controls. Nonselective cortical-hippocampal region lesions disrupted LIRR, whereas selective hippocampal lesions had no detrimental effect on LIRR. Selective entorhinal lesions disrupted LIRR. These findings fit other recent empirical findings and theoretical predictions that some classical conditioning tasks previously thought to depend on the hippocampus depend, rather, on the entorhinal cortex.
\end{abstract}

Prior experiments (Han, Gallagher, \& Holland, 1995; Honey \& Good, 1993; Schmajuk, Lam, \& Christiansen, 1994; Shohamy, Allen, \& Gluck, 2000; Solomon \& Moore, 1975), as well as computational models (Gluck \& Myers, 1993; Schmajuk \& DiCarlo, 1991), have implicated the hippocampal region in mediating stimulus preexposure tasks. A classic preexposure task is latent inhibition(LI), in which preexposure to a conditioned stimulus (CS) alone retards subsequent learning that the CS predicts an unconditioned stimulus (US; Lubow, 1973). A related but less tested preexposure effect is learned irrelevance (LIRR). LIRR is the phenomenon in which uncorrelated presentations of the $\mathrm{CS}$ and the US produce a retardation of conditioning to subsequent CS-US pairings (Baker, 1976; Bennett, Maldonado, \& Mackintosh, 1995; Matzel, Schactman, \& Miller, 1988).

LIRR, which embeds the CS preexposure characterizing LI, has been described as having an even stronger retardation effect than LI. Typically, preexposure to both the CS and the US, uncorrelated with each other, retards subsequent CS-US learning more than does exposure to the CS alone or the US alone (e.g., Bennett et al., 1995).

In a recent behavioral study from our laboratory (Allen et al., 2002), LI and LIRR in eyeblink conditioning were compared in both rabbits and humans. In rabbits, 450 uncorrelated preexposures to a tone and a corneal air puff re-

Correspondence concerning this article should be addressed to M. T. Allen, Department of Psychology, College of Charleston, Charleston, SC 29424 (e-mail: allen@ cofc.edu). tarded acquisition of conditioned responses (CRs) (i.e., LIRR), whereas the same number of CS-alone preexposures failed to produce LI. In that experiment, it was necessary to increase the number of CS-alone preexposures in order to produce LI. On the basis of the number of preexposures used in the present experiment (i.e., 450), we are confident that the preexposure effect we are testing is LIRR that is due to uncorrelated preexposure to the CS and the US, and not simply LI that is due to the preexposure of the CS alone.

There are several theoretical interpretations of the finding that the retardation produced by LIRR is greater than that produced by LI. The simplest explanation is that LIRR simply reflects the sum of CS exposure and US exposure effects (e.g., Baker, 1976) and generates a stronger retardation than either CS-alone or US-alone preexposure. However, there is some evidence suggesting that LIRR exceeds the sum of CS and US exposure effects alone (Matzel et al., 1988). A second explanation is that the lack of correlation between the CS and the US during preexposure further hinders the formation of a CS-US association during the subsequent training phase (e.g., Baker \& Mackintosh, 1979; Bennett et al., 1995; Matzel et al., 1988). A third explanation is that LIRR is a special case of LI (Baker \& Mackintosh, 1979; Bonardi \& Hall, 1996; Hall, 1991). In the work presented here, we address the neural substrates of LIRR, as well as the hypothesized psychologicaland neural mechanisms involved in this form of preexposure effect.

Several theories and computational models have been put forth to explain the psychological and neural mechanisms that may underlie preexposure tasks such as LI and 
LIRR (for a review, see Allen et al., 2002). These theories include the conditioned attention theory (CAT; Lubow, 1989), compression of stimulus representations (Gluck \& Myers, 1993), novelty (Schmajuk, Gray, \& Lam, 1996), and the switching model (Weiner \& Feldon, 1997). Further discussion of these theories that account for both LI and LIRR is found in the General Discussion section.

Several of these theories and models are based on the theory that the hippocampal region is necessary for preexposure effects on subsequent learning. Previous hippocampal lesion studies of preexposure effects in eyeblink conditioning have focused on LI, rather than on LIRR. Aspiration lesions of the hippocampal region disrupted LI in eyeblink conditioning in both rabbits (Solomon \& Moore, 1975) and rats (Schmajuk et al., 1994).

The disruption of LI by aspiration lesions of the hippocampal region was attributed to the removal of the hippocampus. However, although Solomon and Moore's (1975) LI study targeted the dorsal hippocampus, these lesions were nonselective and also damaged overlying cortical structures, including areas of the cingulate, occipital, parietal, prefrontal, and retrosplenial cortices. These aspiration lesions may have also damaged hippocampal afferents from the entorhinal cortex as well. This unintended damage to surrounding cortical regions makes it difficult to conclude that the effects of the hippocampal lesions on LI in eyeblink conditioning reported by Solomon and Moore were due exclusively to hippocampal damage.

Recently, significant improvements in the development of neurotoxic lesion techniques have successfully addressed the issue of damaging the hippocampus without damaging surrounding cortical regions. Jarrard and others have systematically compared the results of lesions produced by different neurotoxins (see Jarrard, 1986, 1989, 1991). The results indicated that ibotenic acid produced the most selective removal of the hippocampus (e.g., Jarrard \& Meldrum, 1993). Experiments designed to compare conventional lesion techniques (e.g., aspiration) and more selective neurotoxic lesions have shown differences in the subjects' ability to acquire certain tasks (e.g., Jarrard, 1993; Jarrard \& Davidson, 1990), suggesting that some behavioral deficits may be dependent on neighboring structures, but not on the hippocampus.

The ibotenic acid selective lesion method has been used to test the effects of selective hippocampal lesions on LI in rats. Honey and Good (1993) found that selective ibotenic acid lesions of the hippocampus in the rat did not disrupt LI for an appetitive task. However, these selective hippocampal lesions did disrupt the context specificity of LI. Han et al. (1995) reported that selective hippocampal lesions did disrupt LI. However, these lesions were made with a mixture of ibotenic acid and NMDA and were tested with a within-subjects version of appetitive conditioning, rather than the standard between-subjects version that is commonly used for LI (Honey \& Good, 1993). Therefore, there is some evidence that although hippocampal region damage disrupts LI, selective hippocampal damage may not have the same effect. Therefore, there is some contro- versy over the role of the hippocampus in LI, at least in these appetitive tasks based on selective lesion techniques.

Recently, we applied the ibotenic acid selective lesion technique (Jarrard, 1989) to rabbits (Shohamy et al., 2000) - specifically, to the dorsal hippocampus and the entorhinal cortex. Selective hippocampal lesions that were comparable to the nonselective hippocampal region lesions of Solomon and Moore (1975) did not disrupt LI in rabbit eyeblink conditioning (Shohamy et al., 2000). However, selective entorhinal lesions disrupted LI in rabbit eyeblink conditioning(Shohamy et al., 2000). Therefore, it appears that for the case of LI, the entorhinal cortex, but not the hippocampus, is necessary.

In the work presented here, we continued this line of research with a related preexposure task, LIRR. Our hypotheses for LIRR were based on our previous selective lesion experiments involving LI in rabbit eyeblink conditioning (Shohamy et al., 2000), as well as on predictions from computational modeling (Gluck \& Myers, 1993; Myers, Gluck, \& Granger, 1995). We hypothesized, on the basis of a previous nonselective hippocampal region lesion study of LI by Solomon and Moore (1975), that nonselective cortical-hippocampal region lesions should disrupt LIRR. However, on the basis of the results of prior studies of LI (Honey \& Good, 1993; Shohamy et al., 2000), we hypothesized that selective hippocampal lesions should have no effect on LIRR. We also hypothesized, on the basis of Shohamy et al.'s findings, that selective entorhinal lesions should disrupt LIRR. In the work presented here, we will discuss the results of tests of the effects of various lesions within the hippocampal region on LIRR.

\section{METHOD}

\section{Subjects}

Forty-four male New Zealand albino rabbits (Oryctolagus cunicu$l u s$ ), weighing over $2.0 \mathrm{~kg}$, were used in this experiment. The rabbits were equally distributed into either an LIRR preexposure or a context preexposure condition. They were also divided into one of the four surgery groups: sham-lesioned controls $(n=12)$, nonselective cortical-hippocampal region lesions $(n=8)$, selective hippocampal lesions $(n=12)$, and selective entorhinal lesions $(n=12)$.

The rabbits were purchased from Covance Laboratories (Princeton, NJ). The rabbits were housed in individual cages in the AAALACaccredited Rutgers University Animal Facility, Newark, NJ. They were given free access to food and water and were maintained on a 12:12-h light:dark cycle, with light on at 7:00 a.m. All the procedures with the rabbits occurred during the light cycle.

\section{Surgery}

All the rabbits were allowed a week of recovery following delivery to the Rutgers Animal Facility before undergoing aseptic lesion surgery. Each rabbit was weighed, and a baseline respiration rate was taken. It was then given a subcutaneous injection of xylazine (Lloyd Laboratories, Shenandoah, IA; $6.0 \mathrm{mg} / \mathrm{kg}$ of body weight). Fifteen minutes later it was given an intramuscular (IM) injection of ketamine (Fort Dodge, Fort Dodge, IA; $60 \mathrm{mg} / \mathrm{kg}$ of body weight). Fifteen minutes later they were given a 1-cc (IM) injection of a ketamine/ xylazine mixture $(2: 1)$. This injection was repeated hourly until the end of the surgical procedure. The rabbit's head was shaved and scrubbed with a betadyne scrub, followed by isopropyl alcohol and 
Table 1

Stereotaxic Coordinates for Selective Dorsal Hippocampal and Selective Entorhinal Lesions

\begin{tabular}{|c|c|c|c|}
\hline \multirow[b]{2}{*}{ Lesion Site } & \multicolumn{2}{|c|}{ Coordinates $(\mathrm{mm})$} & \multirow[b]{2}{*}{$\mathrm{D}-\mathrm{V}$} \\
\hline & $\mathrm{A}-\mathrm{P}$ & $\mathrm{M}-\mathrm{L}$ & \\
\hline Dorsal hippocampus & $\begin{array}{l}-4.0 \\
-5.0 \\
-6.0\end{array}$ & $\begin{array}{l} \pm 3.0 \\
\pm 5.0 \\
\pm 4.0 \\
\pm 7.5 \\
\pm 4.5 \\
\pm 8.0\end{array}$ & $\begin{array}{l}-5.2,-3.2^{*} \\
-5.2^{*},-3.2 \\
-5.2,-3.2^{*} \\
-5.7^{*},-3.5 \\
-5.5,-3.8^{*} \\
-6.5^{*},-4.2\end{array}$ \\
\hline Entorhinal cortex & -5.0 & $\begin{array}{l} \pm 5.5 \\
\pm 6.2 \\
\pm 7.5\end{array}$ & $\begin{array}{l}-19.0,-20.0 \\
-19.0,-20.0 \\
-19.0^{*},-20.0^{*}\end{array}$ \\
\hline
\end{tabular}

Note-Bregma is used as the zero point for the A-P and M-L coordinates, whereas the $\mathrm{D}-\mathrm{V}$ measures are taken from dura. Bregma is $1.5 \mathrm{~mm}$ ventral to lambda. The volume of injection for the hippocampal lesion is .15 or $.10\left(^{*}\right) \mu \mathrm{L}$ at each site, whereas the volume of injection for the entorhinal lesion is . 20 or $.10\left(^{*}\right) \mu \mathrm{L}$ at each site.

betadyne prep solution. The rabbit's respiration rate was monitored at 15-min intervals for the time of the surgical procedures.

The rabbit was placed in a standard stereotaxic head-holder (David Kopf Instruments, Tujunga, CA). An incision was made along the midline of the skull. The skin and muscle were deflected to expose the skull and the bregma and lambda landmarks. Two small holes were drilled in the skull above the hippocampal region to the level of the dura. Two additional small holes were drilled for screws to anchor a dental acrylic headstage. The head was leveled so that lambda was $1.5 \mathrm{~mm}$ higher than bregma.

Selective hippocampal lesion surgery. The rabbits in the selective lesion group received injections of ibotenic acid. Twenty-four bilateral injections of ibotenic acid (10 $\mu \mathrm{g} / \mu \mathrm{L}$, Sigma, St. Louis) were made into the dorsal hippocampus, using a 1.0- $\mu \mathrm{L}$ Hamilton syringe. The stereotaxic coordinates and injection sizes for the dorsal hippocampal ibotenic acid injections are shown in Table 1. For each injection, the needle was lowered to the injection site, and $1 \mathrm{~min}$ was allowed to elapse before injection began. The injection took place over a 2-min period. Following the injection, 2 min were allowed to elapse before the needle was moved to the next injection site. This procedure was repeated for all the injection sites.

Sham lesion surgery. For the rabbits in the sham-operated control group, the surgical procedures were the same as those described for the selective hippocampal lesion group, with the exception that only injections of the vehicle saline solution were made.

Selective entorhinal lesion surgery. For the rabbits in the selective entorhinal lesion group, surgical procedures were the same as those described for the selective hippocampal lesion group, with the exception of the targeting of the ibotenic acid injections to the entorhinal cortex. The stereotaxic coordinates for the 12 bilateral injection sites in the entorhinal cortex are shown in Table 1.

Nonselective cortical-hipp ocam pal lesion surgery. For the rabbits in the nonselective cortical-hippocampal region lesion group, the surgical procedures for the surgery were identical to those for the selective hippocampal surgery, with the exception of the method of hippocampal lesion. The exposed dura was removed to expose the cortex that overlies the hippocampus. The overlying cortex (i.e., the retrosplenial cortex, the frontal cortex, and the parietal cortex) was then aspirated to expose the hippocampus. The dorsal hippocampus was then aspirated to expose the underlying ventricle and midbrain. Gelfoam was placed into the lesion areas to stop bleeding, and bone wax was used to cover the areas of skull removal.

Following the lesion procedures, the holes in the skull were sealed with bone wax. The screws were mounted in the skull, and the exposed skull was covered with dental acrylic. A bolt was attached to the acrylic for the mounting of the eyeblink detector assembly during conditioning. The rabbits were allowed a week for recovery from the surgery prior to the initiation of behavioral conditioning.

\section{Materials}

Each rabbit was restrained in a Plexiglas restraint box in an individual conditioning chamber for eyeblink conditioning. Each chamber contained a speaker, an air hose assembly, and an eyeblink detection system. Presentations of the stimuli and recording of behavioral responses were controlled by a personal computer. The computer housed an interface board (Keithley Metrabyte, Taunton, MA), which triggered a set of relays that controlled the presentation of the tone CS and the air puff US (Chen \& Steinmetz, 1998).

Eyeblinks were monitored with an opto-electronic sensor that consisted of a light-emitting diode (LED) and a phototransistor (for technical details, see Thompson, Moyer, Akase, \& Disterhoft, 1994). The LED emitted a beam of infrared light that was reflected off the cornea, and the reflectance of this beam was converted to a DC voltage by the phototransistor. The eyeblink signal was filtered (between $0.1 \mathrm{~Hz}$ and $1 \mathrm{kHz})$ and amplified $(100 \times)$ by a differential $\mathrm{AC}$ amplifier (A-M Systems, Everett, WA).

When the rabbit closed its eye, the reflectance of the infrared beam changed and was recorded as an eyeblink. Any movement greater than $0.5 \mathrm{~mm}$ recorded during the pre-CS period caused the training trial to be discarded from analysis. A CR was scored if a movement of greater than $0.5 \mathrm{~mm}$ was recorded during the CS period (i.e., the time between CS and US onsets). An unconditioned response was scored if a movement of greater than $0.5 \mathrm{~mm}$ was recorded following the onset of the US. Each trial's behavioral record was displayed on the computer screen. The computer stored and analyzed the behavioral data and delivered a printed data summary for each 50-trial block.

\section{Stimuli}

The CS was a 450-msec, $90-\mathrm{dB}, 1000-\mathrm{Hz}$ tone delivered from a speaker located in front of the rabbit's face. The US was a 50-msec, 3 -psi corneal air puff delivered via a rubber hose attached to the eyeblink detector assembly and aimed at the rabbit's cornea.

\section{Design and Procedure}

Adaptation. Prior to preexposure and training, all the rabbits were adapted to the conditioning chamber and restraint box for two daily sessions. On the 1st day of adaptation, the rabbit was placed in the restraint box in the conditioning chamber for a half hour. On the 2nd day of adaptation, the rabbit was placed in the restraint box in the conditioning chamber, with the eyeblink detector aimed at its cornea for $45 \mathrm{~min}$.

Preexposure phase. In the LIRR preexposure condition, the rabbits were preexposed to pseudorandom presentations of the tone CS and the air puff US. The preexposure trials were $20 \mathrm{sec}$ in length, with a 1-sec intertrial interval (ITI). The tone and air puff were pseudorandomly separated by 5 or $10 \mathrm{sec}$ in either a forward or a backward fashion (i.e., on half the trials the tone preceded the air puff, and on half it followed the air puff). In this way, the tone had an equal probability of occurring either prior to or after the air puff, but never in close temporal proximity. This interval of temporal separation of the tone and the air puff was outside the temporal limit for rabbit eyeblink conditioning (Gormezano, Kehoe, \& Marshall, 1983), so no association would be formed between the tone and the air puff. This pattern of stimulus presentations was used so that there would be no learning of a causal relationship between the tone and the air puff during the preexposure phase.

The LIRR-preexposed rabbits received 100 daily trials of the random CS and US for four and a half daily sessions, for a total of 450 preexposures. On the 5 th day of LIRR preexposure, they received 50 LIRR preexposure trials, followed immediately by 50 acquisition trials of tone-air-puff training. These training parameters were based on the LI study of Solomon and Moore (1975). 


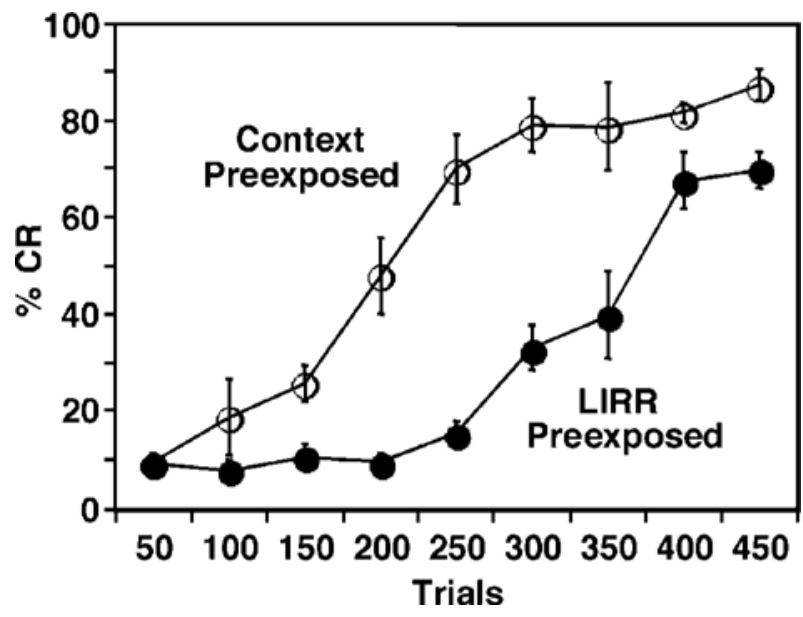

Figure 1. Behavioral results from the sham-operated control groups. The learning curves (graphed as percentage of conditioned responses [CRs]) are shown for the context-preexposed group (open circles) and the learned irrelevance (LIRR) preexposed group (closed circles). Sham-lesioned rabbits in the LIRR-preexposed condition were significantly retarded in acquiring CRs, as compared with those in the context-preexposed group. This is a demonstration of LIRR.

In the context preexposure condition, the rabbits received the same number of preexposure trials as the LIRR-preexposed group. However, these were blank trials in which no stimuli were presented. In this fashion, the context-preexposed group received the same amount of preexposure to the conditioning context as the LIRRpreexposed group, but with no learning about the stimuli.

Acquisition phase. Starting with the second half of the 5th day of preexposure, the tone and the air puff were paired in a standard delay paradigm. All the rabbits received forward-paired presentations of a 450-msec, $1000-\mathrm{Hz}, 95-\mathrm{dB}$ tone, which coterminated with a 50msec, 3-psi corneal air puff. The interstimulus interval was $400 \mathrm{msec}$. The ITI was a pseudorandom number that was between 25 and $35 \mathrm{sec}$. The rabbits received a total of 450 paired trials over four and a half daily training sessions. Training criterion was defined as eight CRs out of nine consecutive trials.

Histology. Following completion of conditioning, all the rabbits were overdosed by an intravenous injection of pentobarbital (Henry Schein, Port Washington, NY; $324 \mathrm{mg} / \mathrm{kg}$ ) into the marginal ear vein. All the rabbits were then perfused via the ascending aorta with $1 \mathrm{~L}$ of a $0.9 \%$ saline solution followed by $1 \mathrm{~L}$ of a $10 \%$ formalin solution. The brain was then removed and preserved in a $30 \%$ sucrose/ $10 \%$-formalin solution for a week. Serial coronal 80 -micron sections through the hippocampal region were taken, mounted on slides, and stained with cresyl violet.

Data analysis. Repeated measures analyses of variance of the behavioral data were done for each 50-trial block. Pairwise comparisons were planned for the LIRR preexposure condition and the contextpreexposed condition for each of the four lesion groups (i.e., sham, nonselective cortical-hippoca mpal region, selective hippocampal, and selective entorhinal lesions). In addition, LIRR-preexposed rabbits in each of the lesion conditions were also compared with LIRRpreexposed rabbits in the sham-lesioned condition to test for lesion effects on the LIRR preexposure effect.

The histological results were analyzed for the size and location of the hippocampal lesions, which were assessed by viewing the slice under a microscope and drawing the lesions, based on neuronal loss, onto a template of a corresponding sham-lesioned hippocampus. The location and extent of our selective dorsal hippocampal lesions were analyzed by comparing the area of the lesion to the area of the corresponding sham-lesioned (i.e., nondamaged) hippocampal sections.

\section{RESULTS}

\section{Sham-Lesioned Controls}

All the sham-lesioned rabbits met the training criterion of eight CRs out of nine consecutive trials. All the shamlesioned rabbits exhibited more CRs as training progressed across session $[F(8,80)=57.2, p<.001]$. The learning curves for the sham-operated rabbits are shown in Figure 1.

The sham-lesioned rabbits in the LIRR-preexposed condition exhibited significantly fewer CRs than did those in the context-preexposed condition $[F(1,10)=11.163$, $p<.01]$. The sham-lesioned rabbits in the LIRR-preexposed condition were also slower to acquire CRs, as compared with those in the context-preexposed condition, as was indicated by a significant group $\times$ session interaction $[F(8,80)=4.375, p<.001]$.

\section{Nonselective Cortical-Hippocampal Lesion Groups}

Histology. Figure 2A shows minimal (gray) and maximal (black) cortical-hippocampalregion lesions. These lesions removed the dorsal hippocampus and the overlying cortex (i.e., portions of the retrosplenial cortex, the frontal cortex, and the parietal cortex). Our nonselective corticalhippocampal lesions were comparable and even exceeded the size and extent of the nonselective hippocampal aspiration lesions from Solomon and Moore's (1975) LI experiment, as shown in Figure 2C.

Conditioning. All the nonselectivecortical-hippocampal lesioned rabbits met the training criterion of eight CRs out of nine consecutive trials. All the nonselective corticalhippocampal lesioned rabbits exhibited more CRs as training progressed across sessions $[F(8,48)=2.78, p<$ $.05]$. The learning curves for the nonselective corticalhippocampal region lesion groups are shown in Figure 3.

Unlike in the sham-lesioned controls, the corticalhippocampal region lesioned rabbits exhibited no significant differences in CRs between the LIRR-preexposed and the context-preexposed conditions $[F(1,6)=0.428, p>.5]$. There was no significant group $\times$ session interaction $[F(8,48)=1.057, p>.4]$. A nonsignificant trend was still present, so that the cortical-hippocampal region lesioned rabbits in the LIRR-preexposed condition tended to exhibit fewer CRs than did those in the context-preexposed condition. The nonselectivecortical-hippocampalregion lesioned rabbits in the LIRR-preexposed condition exhibited significantly more CRs than did the sham-lesioned rabbits in the LIRR-preexposed condition $[F(1,16)=10.97, p<.01]$. There were no significant interactions between group, lesion, and session for the sham-lesioned and cortical hippocampal lesion groups $(p>.05)$. Thus, nonselective cortical-hippocampal region lesions disrupted LIRR.

\section{Selective Hippocampal Lesion Groups}

Histology. Figure 2B shows the minimal and maximal selective hippocampal lesions obtained. These maximal lesions (shown in black) included the CA1-CA3 cell fields and the dentate gyrus of the dorsal hippocampus. 

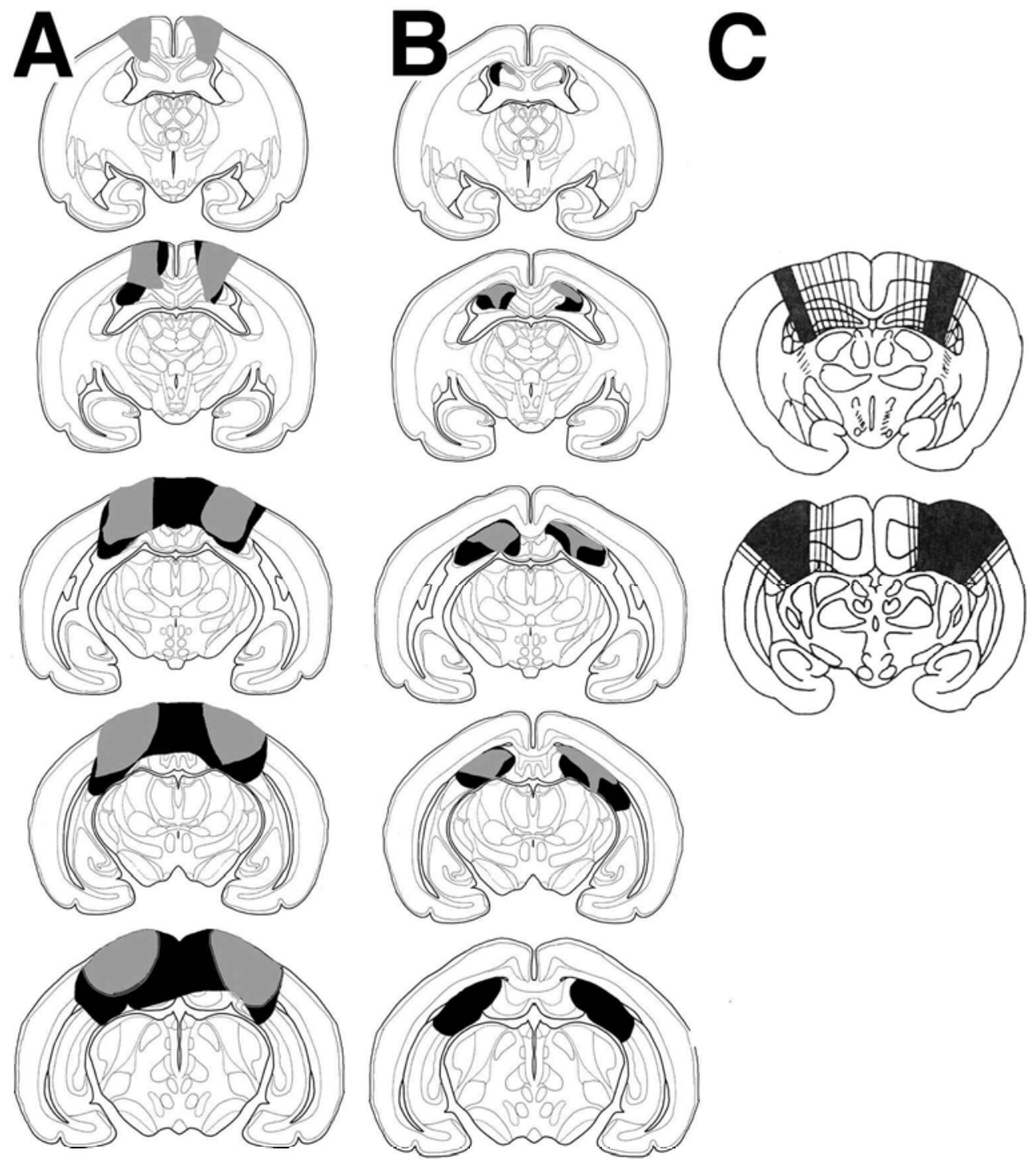

Figure 2. Comparisons of nonselective cortical hippocampal and selective hippocampal lesions with the reported histology of Solomon and Moore (1975). The size and extent of both our nonselective cortical hippocampal region and our selective hippocampal lesions are comparable to those of Solomon and Moore in panel $C$. The brain schematics are adapted from Urban and Richard (1972). (A) Histology of the nonselective cortical-hippocampal region lesions, with the minimal (gray) and maximal (black) lesion sizes for the cortical-hippocampal ablation lesions. The top section is the most rostral. Each subsequent section is $1 \mathrm{~mm}$ caudal to the previous section. This figure is adapted from the stereotaxic atlas of Urban and Richard. (B) Histology of the selective ibotenic acid hippocampal lesions, with the minimal (gray) and maximal (black) lesion sizes for the selective hippocampal lesions. The top section is the most anterior. Each subsequent section is $1 \mathrm{~mm}$ posterior to the previous section. (C) The histology of nonselective hippocampal region lesions from Solomon and Moore's study of latent inhibition.

Minimal lesions (shown in gray) were isolated to the CA1 and CA3 cell fields, with some minimal damage to the dentate gyrus of the dorsal hippocampus. Maximal lesions removed all the cell fields in the dorsal hippocampus.

As was previously discussed, the hippocampal lesions in the LI study of Solomon and Moore (1975) were nonselective aspiration lesions that targeted the dorsal hippocampus. We compared the location and extent of the minimal (gray area) and maximal (black area) lesions for our selective hippocampal lesions (Figure 2B) with the histology presented by Solomon and Moore for their hippocampal lesion LI study (Figure 2C). As can be observed, the location and extent of our selective lesions matched those of Solomon and Moore. In both cases, the majority of damage was to the dorsal hippocampus, with some damage to the more caudal and ventral portions of the hippocampus, but with no damage to the ventral hippocampus in the rostral portion. A photomicrograph of a repre- 


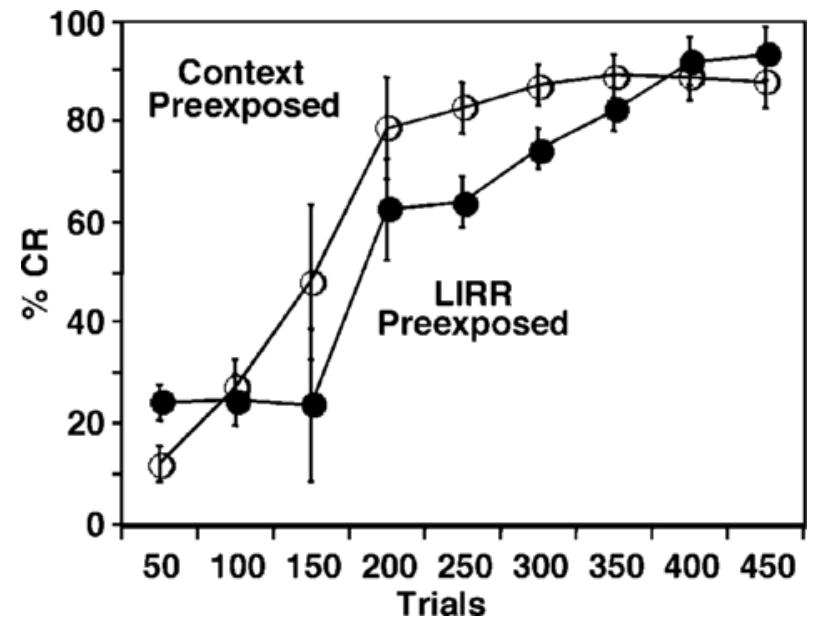

Figure 3. Behavioral results for the cortical-hippocampal ablation lesion groups. The learning curves (graphed as percentage of conditioned responses [CRs]) are shown for the context-preexposed group (open circles) and the learned irrelevance (LIRR) preexposed group (closed circles). Nonselective cortical-hippocampalregion-lesioned rabbits exhibited no significant differences in conditioned responding between the LIRR-preexposed condition and the context-preexposed condition. Thus, LIRR was disrupted following nonselective cortical-hippocampal lesions.

sentative sham-lesioned control hippocampus and a selective ibotenic acid hippocampal lesion is shown in Figure 4.

Conditioning. All the selective hippocampal-lesioned rabbits met the training criterion of eight CRs out of nine consecutive trials. All the selective hippocampal-lesioned rabbits exhibited more CRs as training progressed across sessions $[F(8,80)=42.0, p<.001]$. The learning curves for the selective hippocampal lesion groups are shown in Figure 5.

Unlike the cortical-hippocampalablation group, the selective hippocampal-lesioned rabbits exhibitedLIRR. The selective hippocampal-lesioned rabbits in the LIRRpreexposed condition exhibited significantly fewer CRs than did those in the context-preexposed condition $[F(1,10)=$ $5.57, p<.05]$. Overall, the selective hippocampallesioned rabbits in the LIRR-preexposed condition were slower to acquire CRs, as compared with those in the context-preexposed condition, as was indicated by a significant session $\times$ group interaction $[F(8,80)=3.017, p<.01]$.

Unlike our results following the nonselective corticalhippocampal region lesions, the selective hippocampallesioned rabbits in the LIRR-preexposed condition did not exhibit any significant differences in CRs, as compared with sham-lesioned rabbits in the LIRR-preexposed condition $[F(1,20)=0.945, p>.34]$. There was a significant interaction of group and lesion $[F(1,20)=28.196, p<$ $.02]$, as well as a significant interaction of session and group $[F(8,160)=6.107, p<.001]$. There was no other significant interaction $(p>.05)$. Thus, selective hippocampal lesions did not disrupt LIRR.

\section{Selective Entorhinal Lesioned Groups}

Histology. Figure 6 shows the minimal and maximal selective ibotenic acid entorhinal lesions obtained. Maximal lesions (shown in black) damaged the majority of the entorhinal cortex. Minimal lesions (shown in gray) were isolated to the medial half of the entorhinal cortex. A photomicrograph of a representative selective ibotenic acid entorhinal lesion is shown in Figure 7.

Conditioning. All the selective entorhinal-lesioned rabbits met the training criterion of eight CRs out of nine consecutive trials. All the selective entorhinal-lesioned rabbits exhibited more $\mathrm{CRs}$ as training progressed across sessions $[F(8,80)=35.862, p<.001]$. The learning curves for the selective entorhinal lesion groups are shown in Figure 8.

The results for the selective entorhinal-lesioned rabbits were similar to those obtained with the cortical-hippocampal
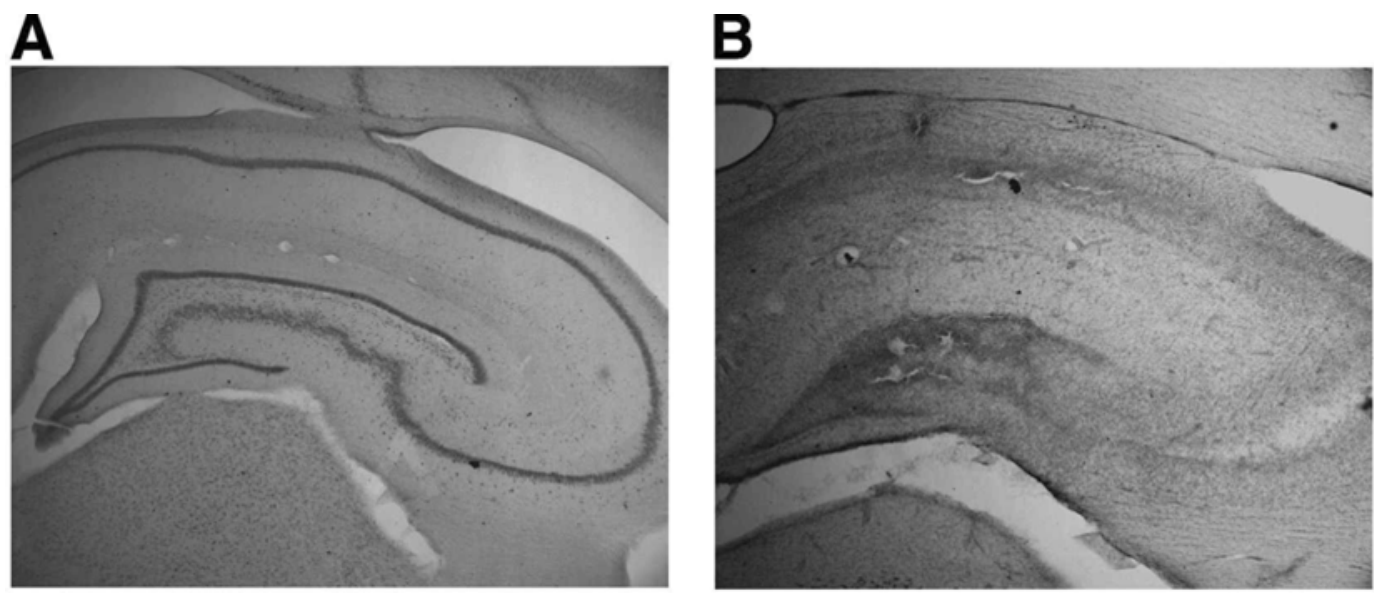

Figure 4. Photomicrographs of selective ibotenic acid hippocampal lesions. (A) A photomicrograph (magnification $40 \times$ ) of a representative sham-lesioned dorsal hippocampus. (B) A photomicrograph (magnification $40 \times$ ) of a representative selective hippocampal lesion. Note the complete loss of the cell fields for the dorsal hippocampus, as compared with the thick, darkly stained cell field in the sham-lesioned hippocampus in Figure 4A. 


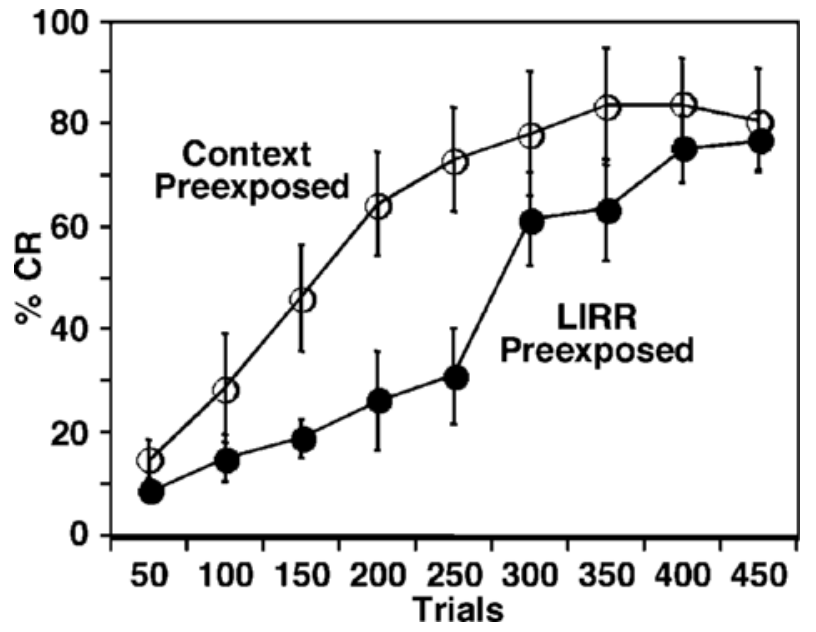

Figure 5. Behavioral results for the selective ibotenic acid hippocampal lesion groups. The learning curves (graphed as percentage of conditioned responses [CRs]) are shown for the contextpreexposed group (open circles) and the learned irrelevance (LIRR) preexposed group (closed circles). Selective hippocampallesioned rabbits in the LIRR-preexposed condition were significantly retarded in CRs, as compared with those in the contextpreexposed condition. Thus, LIRR was intact following a selective hippocampal lesion.

ablation lesions. Selective entorhinal-lesioned rabbits exhibited no significant differences in conditioned responding between the LIRR-preexposed and the context-preexposed conditions $[F(1,10)=1.25, p>.28]$. There was a significant session $\times$ group interaction $[F(8,80)=3.219, p<$ .005].

There was a lesion effect such that the selective entorhinal-lesioned rabbits in the LIRR-preexposed condition exhibited more $C R s$ than did the sham-lesioned rabbits in the LIRR-preexposed condition $[F(1,20)=8.162$, $p<.05]$. There was no significant lesion $\times$ group interaction $(p>.09)$. There was a significant session $\times$ lesion interaction $[F(8,160)=5.20, p<.001]$. There was also a significant session $\times$ group $\times$ lesion interaction $[F(8,160)=3.42, p<.01]$. Thus, selective entorhinal lesions disrupted LIRR.

\section{DISCUSSION}

Overall, uncorrelated preexposures to the tone CS and the air puff US in sham-lesioned rabbits retarded acquisition of CRs, as compared with context-preexposed rabbits. This was a demonstration of the LIRR preexposure effect. The same number of CS-alone preexposures failed to produce LI in a previous study (Allen et al., 2002). All the sham-lesioned rabbits were able to reach asymptotic responding within 450 conditioning trials. Therefore, LIRR preexposure did not block the acquisition of CRs but only retarded its development.

Since we were successful in demonstrating LIRR in rabbit eyeblink conditioning, using sham-lesioned rabbits, our next step was to compare the effects of nonselective and selective hippocampallesions on LIRR. Nonselective cortical-hippocampal region lesions that included the overlying cortex disrupted LIRR, whereas comparable selective hippocampal lesions had no effect on LIRR. These results were consistent with previous findings demonstrating that nonselective cortical-hippocampal region lesions impaired LI in eyeblink conditioning in rabbits (Solomon \& Moore, 1975) and in rats (Kaye \& Pearce, 1987; Schmajuk et al., 1994). The finding that selective hippocampal lesions did not disrupt LI was also consistent with prior studies in rats (Honey \& Good, 1993) and in rabbit eyeblink conditioning (Shohamy et al., 2000).

Although our nonselective and selective hippocampal lesions were not complete (i.e., they both spared areas of the ventral hippocampus), our nonselective hippocampal

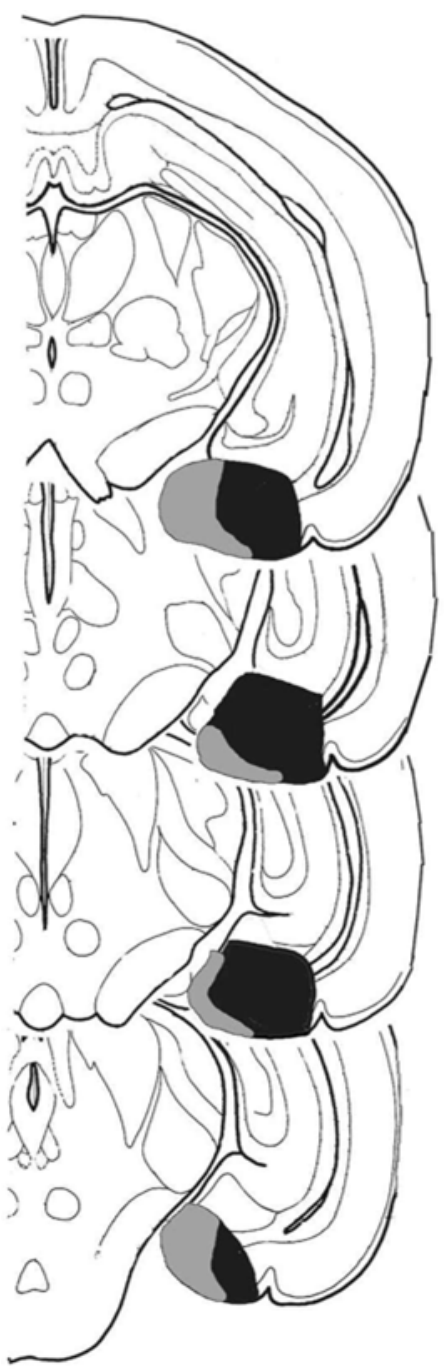

Figure 6. Histology for the selective ibotenic acid entorhinal lesions, with the minimal (gray) and maximal (black) selective entorhinal cortical lesions. The lesions were bilateral, but for simplicity this figure shows only the minimal and the maximal lesions mapped onto a unilateral view. The brain schematics are adapted from Urban and Richard (1972). 


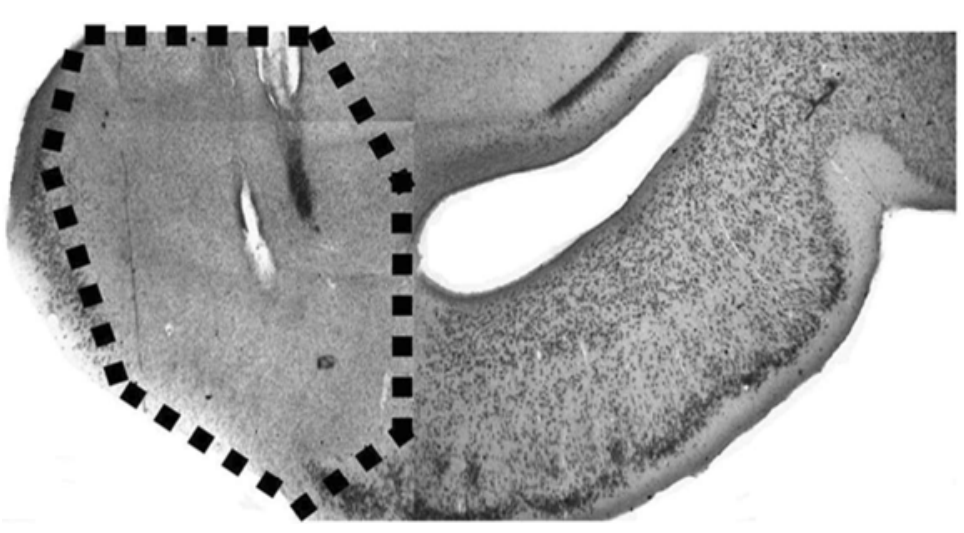

Figure 7. Photomicrograph of selective ibotenic acid entorhinal lesions. A photomicrograph (magnification $40 \times$ ) of a representative selective entorhinal lesion is shown. The area of the lesion of the entorhinal cortex is marked by the dashed lines.

lesions were comparable in size, location, extent, and effectiveness in disrupting a preexposure task to the nonselective aspiration lesions of Solomon and Moore's (1975) LI study. In addition, our lesions resulted in the differential finding that a nonselective dorsal hippocampal region lesion disrupts LIRR, whereas a selective dorsal hippocampal lesion does not disrupt LIRR. Therefore, our overall finding from these two types of lesions to the dorsal hippocampal is the differential effect of nonselective and selective hippocampal lesions on LIRR.

The finding that the hippocampus proper is not necessary for the LIRR effect raised the question of what nonhippocampal structure within the hippocampal region may be responsible for the nonselective hippocampal lesion effect. The possible candidate that we tested was the entorhinal cortex. Among selective entorhinal-lesioned rabbits, LIRR-preexposed rabbits were not significantly different from context-preexposed controls. This finding, along with the selective hippocampal lesion results reported here, demonstrates that it is possible to differentiate between selective hippocampal and selective entorhinal lesion effects, at least for a preexposure task such as LIRR.

Figure 9 shows our results replotted to compare the four lesion groups (sham, nonselective cortical hippocampal, selective hippocampal, and selective entorhinal) for the LIRR preexposure condition. Overall, it is apparent that LIRR preexposure resulted in a retardation of acquisition in sham-lesioned and selective hippocampal-lesionedrabbits, but not in nonselective cortical-hippocampal region lesioned or selective entorhinal-lesioned rabbits.

\section{GENERAL DISCUSSION}

Our overall findings from this experiment demonstrate that whereas the hippocampus proper is not necessary for LIRR to occur, the entorhinal cortex is essential. These findings fit with prior results obtained from similar lesion experiments in our laboratory, done with LI (Shohamy et al., 2000). Together, these two studies provide evidence for an entorhinal cortical role in preexposure effects such as LI and LIRR that is distinct from the role of the hippocampus.

Lesions of the entorhinal cortex and the perirhinal and parahippocampal cortices have been found to affect other learning paradigms previously considered "hippocampal" tasks. These tasks include working memory tasks, such as delayed nonmatching-to-sample (Suzuki, Zola-Morgan, Squire, \& Amaral, 1993), spatial tasks, such as the Mor-

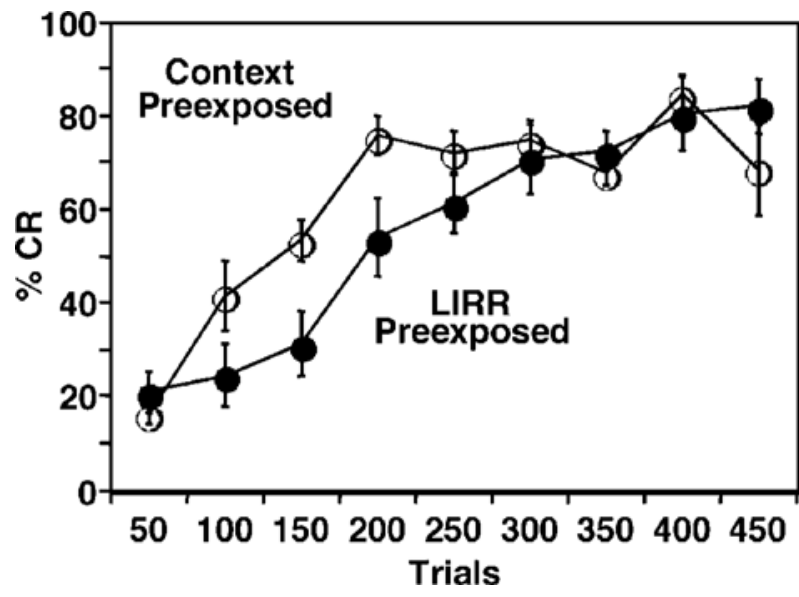

Figure 8. Behavioral results for the selective ibotenic acid entorhinal cortical lesion groups. The learning curves (graphed as percentage of conditioned responses [CRs]) are shown for the context-preexposed group (open circles) and the learned irrelevance (LIRR) preexposed group (closed circles). Selective entorhinal-lesioned rabbits in the LIRR-preexposed condition were not significantly retarded in acquiring CRs, as compared with selective entorhinal-lesioned rabbits in the context-preexposed condition. That is, LIRR preexposure was disrupted following a selective entorhinal cortical lesion. 


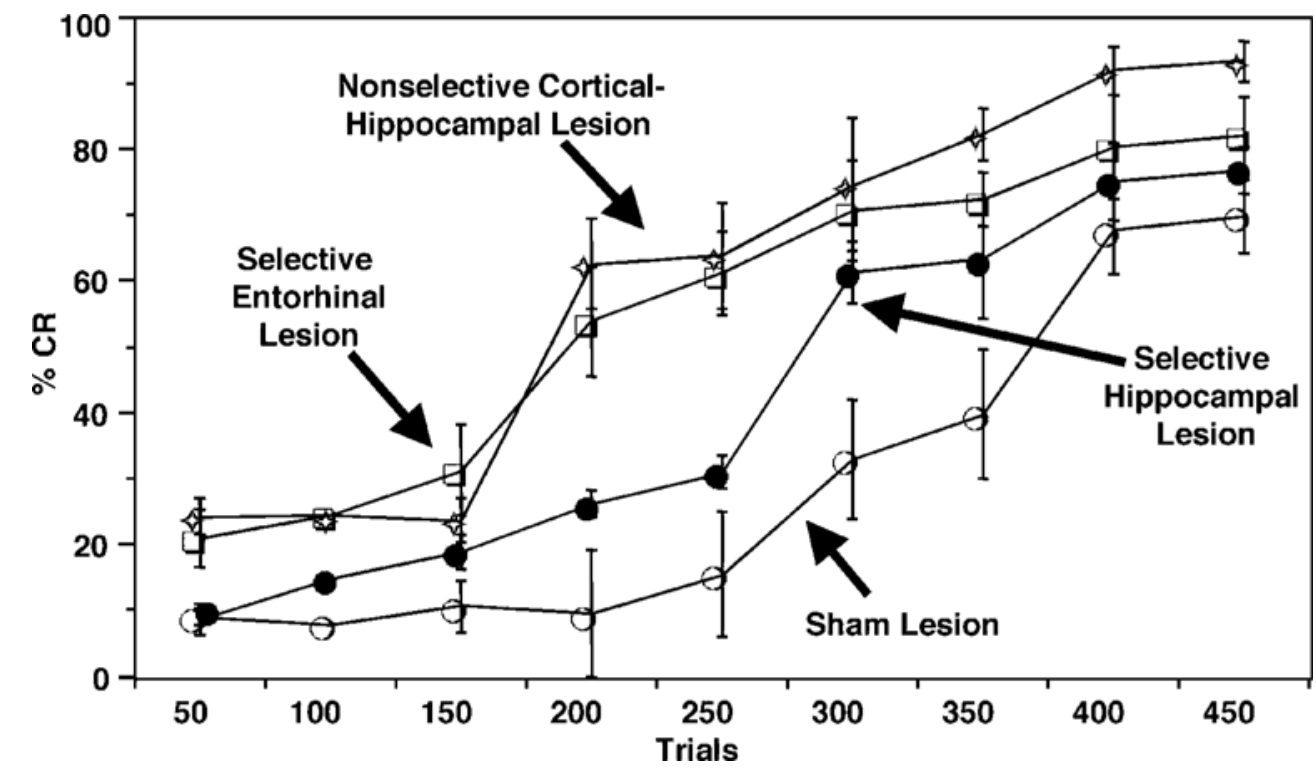

Figure 9. Summary of the effects of learned irrelevance (LIRR) preexposure for the four lesion groups tested. LIRR preexposure resulted in a retardation of acquisition for sham-lesioned and selective hippocampal-lesioned rabbits, but not for nonselective cortical-hippocampal-region-lesioned or selective entorhinal-lesioned rabbits. Overall, these results indicate that the hippocampal region and, more specifically, the entorhinal cortex, but not the hippocampus itself, are necessary for the LIRR.

ris water maze (Glasier, Janis, Roof, \& Stein, 1999) and the radial arm maze (Cho \& Kesner, 1996; Glasier, Janis, \& Stein, 1997; Otto, Wolf, \& Walsh, 1997), and working memory discrimination tasks (Yee \& Rawlins, 1998).

One study of particular interest to our work on rabbit eyeblink conditioning is a test of the effects of cytotoxic entorhinal lesions in rats on a working memory discrimination task involving complex three-dimensional objects (Yee \& Rawlins, 1998). Entorhinal lesions impaired performance on tasks in which the correct response required that a response be made to an object that had recently been nonrewarded. In addition, entorhinal lesions were found to enhance reversal learning following a simple discrimination task. This finding is of interest to us because of the hippocampal lesion effects seen in reversal of a discrimination task in rabbit eyeblink conditioning. Berger and Orr (1983) found that nonselective electrolytic lesions of the hippocampal region did not affect a simple two-tone discrimination but did impair reversal. Specifically, there was an overresponding to the previous CS + tone. In other words, the rabbit could not learn to inhibit responding to a previously reinforced cue. The finding of Yee and Rawlins is interesting in that they found that entorhinal lesions actually enhanced reversal learning. One interpretation of this finding is that whereas a hippocampal lesion impairs reversal, an entorhinal lesion enhances it. This finding should be investigated further in eyeblink conditioning experiments.

Yee and Rawlins (1998) also found that cytotoxic entorhinal lesions impaired performance on trials in which a correct response must be made to an object that had pre- viously been unrewarded. This finding is very similar in nature to preexposure tasks such as LIRR or LI. In preexposure tasks, acquisition is normally slower to stimuli that have previously been exposed without reinforcement. In our results, however, a selective entorhinal lesion disrupted this effect, and the rabbits learned as if they had not been preexposed. Yee and Rawlins found that rats were impaired at properly responding on this type of task. The difference in the effects of an entorhinal lesion on these tasks may be due to differences in the requirements of the tasks; however, in both cases, entorhinal lesions affected tasks involving responding to previously irrelevant stimuli.

\section{The Role of Context in Preexposure Effects}

An issue that was not addressed by the work presented here but would be of interest for future experiments is the effect of context on preexposure effects. Prior work by Honey and Good (1993) indicated that selective hippocampal lesions disrupt the contextual specificity of LI, but not LI itself. Holt and Maren (1999) found that muscimol inactivation of the dorsal hippocampus affects only the context specificity of LI, but not the expression of LI. Therefore, it appears that the hippocampus is necessary for the contextual specificity of LI, but not for the acquisition and expression of LI. It is unclear whether LIRR is also contextually specific. If it is the case that LIRR is context specific, it is probable that selective hippocampal lesions would disrupt this.

Another issue involving context and preexposure effects comes from a recent report (Rogers, Katz, Gorin, \& Steinmetz, 2001) that suggested that LI may involve both a 
retardation effect owing to $\mathrm{CS}$ preexposure and a facilitation effect owing to contextual preexposure in the control group.

Future work should test the contextual specificity of LIRR. It may be that LIRR would not be contextually specific. Since both the CS and the US are preexposed, learning of the uncorrelated relationship between these stimuli may be more generalizable to other contextual situations than would the learning about the CS alone during LI.

\section{Hypothesized Mechanisms for Preexposure Effects}

Several theories and computational models have been put forth to explain the mechanisms that underlie preexposure tasks such as LI. Using these theories, we are able to interpret and predict an LIRR effect that follows from LI.

CAT, put forth by Lubow (1989), states that nonreinforced exposures to a stimulus retards subsequent acquisition of CRs to that stimulus, owing to the animal's learning not to attend to the unreinforced CS. CAT also can account for LIRR. Lubow (1989) stated that CAT would predict a more profound retardation effect from a noncontingent preexposure of the CS and US, as compared with that observed following CS-alone (LI) preexposures.

Lubow (1989) also put forth an explanation of greater retardation that was based on the salience of the preexposed cues. Schnur and Lubow (1976) found that more retardation was found with stronger CSs than with weaker CSs. Lubow (1989) predicted that the presentation of the more intense and aversive US during unreinforced preexposure with the CS (as would be the case for LIRR) would also result in a greater retardation than that for LI following CS-alone presentations.

Gluck and Myers (1993) put forth a computationalmodel that proposes a neural mechanism for LI that can account for LIRR, as well as for LI. Their original model simulated only LI but, more recently, Gluck and Myers (2001) repeated these simulations for LIRR. Gluck and Myers's (1993) model proposes that the hippocampal region forms stimulus representations during learning. One mechanism that is used in forming stimulus representations is redundancy compression, in which a single representation for two stimuli is formed when the two stimuli either co-occur or predict the same behavioral outcome.

In the case of LI preexposure, the CS and the context co-occur with no behavioral outcome. Therefore, the CS and the context are compressed into a single representation. Later in training, when the CS must be differentiated from the context as the predictor for the US, this compression must be uncompressed. Therefore, animals that have undergone CS preexposure are slower to learn that the CS predicts the US than are context preexposed controls.

In the case of LIRR, the CS and the US both co-occur with the context, but not in temporal proximity to each other. Therefore, during LIRR preexposure, the CS and the context are compressed, and the US and the context are compressed, but the CS and the US are not compressed together. If the CS and the US were compressed during
LIRR preexposure, one would expect LIRR preexposure to result in a facilitation of CS-US learning, since the association was formed prior to training. Therefore, to overcome the effects of LIRR preexposure, both the CS and the US must be uncompressed from the context in order to form the CS-US association. It is interesting to note, in the simulations of LIRR and LI from Gluck and Myers's (1993) model, that LIRR preexposure produces a larger retardation effect than does LI, because learning following preexposures to the CS and the US requires more decompression than does preexposure to the CS alone. It is also interesting to note that in both cases, the difference in the learning curves are later in training, during asymptotic levels of performance of CRs.

A hippocampal model put forth by Schmajuk et al. (1996) and Buhusi, Gray, and Schmajuk (1998) proposes that preexposure effects such as LI are due to a mechanism based on novelty. Novelty in their model is much like the idea of error correction in the Rescorla-Wagner model (i.e., the difference between the expected US and the actual presentation of the US). In their model, the fastest learning occurs when novelty is high, and slower learning occurs when novelty is low. Therefore, when the CS is not novel (i.e., following CS preexposure), CS-US learning is slower than when the CS is novel (i.e., following context preexposure).

The idea of novelty can also explain LIRR, as well as LI. Following context preexposure, both the CS and the US are novel, but the context is not novel at the start of the training phase. Therefore, learning should be rapid. Following CS preexposure for LI, only the US is novel at the start of the training phase (i.e., the subjects have already experienced the CS and context in preexposure). Therefore, learning should be slower, as compared with contextpreexposed controls. However, following uncorrelated CS and US preexposures for LIRR, the subjects have been preexposed to the context, the CS, and the US, and the only novelty at the start of training is the pairing of the CS and the US. Therefore, LIRR results in less novelty and slower training, as compared with both context-preexposed and CS-preexposed subjects.

A related theory, based in part on the mechanism of Schmajuk and Moore's (1988) attentional-associative model, is the switching theory of Weiner and Feldon (1997). The switching theory proposes that retardation following CS-alone preexposures occurs because there is a conflict between the nonreinforced presentations of the CS during preexposure and the same cue's being reinforced in training. In preexposure, the animal learns that the CS predicts no event but, in training, must learn that the CS predicts the US.

We can interpret the switching model as supporting LIRR, as well as LI. This is due to the fact that there would be less associability between the CS and the US following LIRR, since the animal has explicitly learned that the CS and the US occur separately. The animal would learn that the CS signals no event and that the US occurs with no 

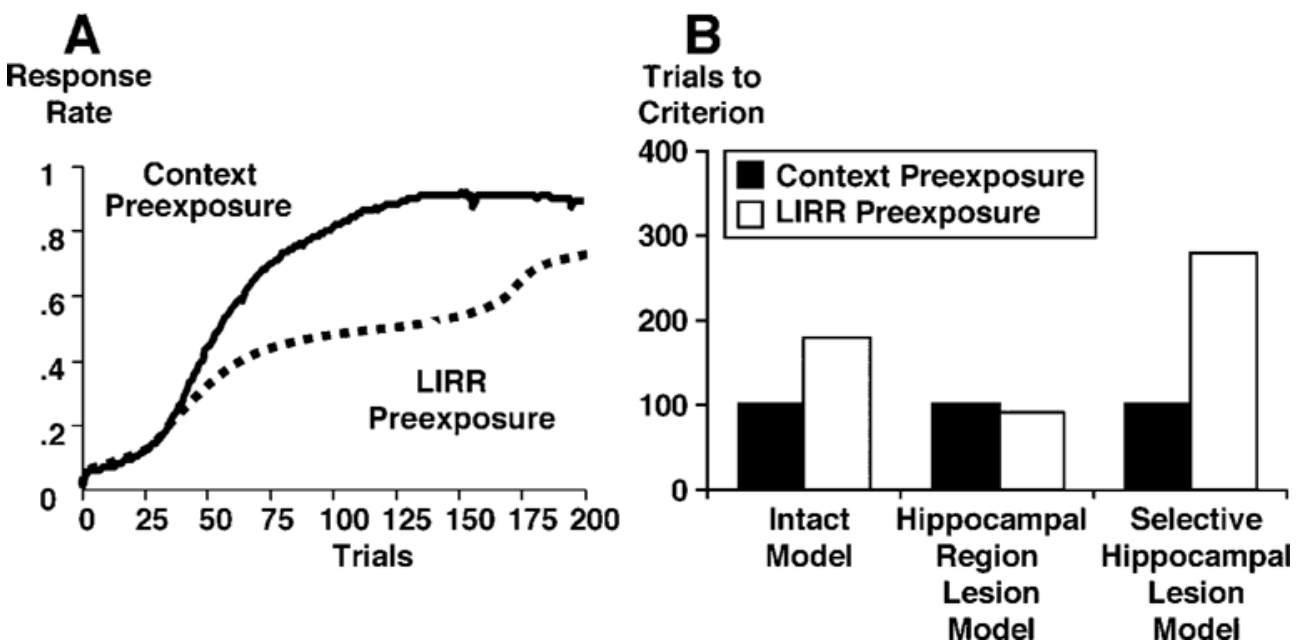

Figure 10. Computational model simulations of learned irrelevance (LIRR) from Gluck and Myers's (1993) and Myers, Gluck, and Granger's (1995) models of hippocampal region and entorhinal function. (A) Simulations of the learning curves following either uncorrelated conditioned stimulus (CS) and unconditioned stimulus (US) preexposures or context-alone preexposures. Uncorrelated CS and US preexposures produce a retardation of acquisition to subsequent paired presentations of the CS and the US (i.e., LIRR). (B) Simulations of the effects of hippocampal region lesions and selective hippocampal lesions on LIRR (graphed as trials necessary to reach a criterion of eight conditioned responses [CRS] out of nine consecutive trials). The intact model produces LIRR as an increased number of trials necessary to reach criterion following uncorrelated CS and US preexposures, as compared with context-alone preexposure. The hippocampal-region-lesioned model that lacks both hippocampal and entorhinal mechanisms disrupts LIRR so that there is no difference between the effects of uncorrelated CS and US preexposures and of context-alone preexposure. Finally, a selective hippocampal lesion model that lacks the hippocampal mechanism of differentiation but leaves intact the entorhinal cortex mechanism of compression does not disrupt LIRR.

signal. This would lead to a lower associability than would occur following learning that the CS signals no event.

\section{Theories of Entorhinal Cortical Function}

In addition to explaining the psychologicalmechanisms that may be involved in preexposure tasks such as LIRR and LI, some of these theories also hypothesize the neural substrates within the hippocampal region that are necessary for these preexposure tasks.

Since the original publication of the Gluck and Myers (1993) and Myers et al. (1995) studies, simulations of various lesion effects on LI have been produced for LIRR as well (Gluck \& Myers, 2001). The simulations of the basic LIRR effect are shown as learning curves in Figure 10A and as trials to criterion in Figure 10B.

The Gluck-Myers (1993) model originally simulated the effects of a hippocampal region lesion in disrupting LI (Kaye \& Pearce, 1987; Schmajuk et al., 1994; Solomon \& Moore, 1975). This prediction has since been made for LIRR, as is shown in Figure 9B. Myers et al.'s (1995) model predicted that selective hippocampal lesions that spare the entorhinal cortex should spare LI and LIRR, as is shown in Figure 10B. Implicit in this model is the prediction that an entorhinal cortical lesion that disrupts the mechanism of redundancy compression would disrupt preexposure tasks such as LI and LIRR. However, at pres- ent, this selective entorhinal lesion model that leaves the hippocampus intact has yet to be developed.

Eichenbaum, Otto, and Cohen (1994) have theorized that the parahippocampal region (including the entorhinal cortex) serves as an intermediate-term buffer, whereas the hippocampal formation mediates the representational processes that allow for generalization of learned associations to novel situations. Although intermediate-term memory differs from the stimulus-stimulus compression function for entorhinal function proposed by Myers et al. (1995), the two functions are complementary. In real-world situations, events that conceptually co-occur may not actually co-initiate or coterminate; intermediate storage of recent events might allow the system to cluster stimuli that reliably occur close together in time. Conversely, the intermediate-term buffer probably has to perform clustering to reduce the information passing through it.

Bunsey and Eichenbaum (1993) also have suggested that the parahippocampal region (including the entorhinal cortex) mediates the fusion of co-occurring or nearly coincident stimuli. This process is functionally analogous to the redundancy compression put forth by Myers et al. (1995). It is interesting to note that Bunsey and Eichenbaum's fusion theory was derived from behavioral observations of paired associate learning in intact, hippocampallesioned, and parahippocampal-lesionedanimals. However, 
Myers et al.'s compression theory was based on an integration of both physiologically based and behaviorally based computational models of hippocampal region function. The convergence of these two diverse empirical and computational approaches to theory development is hopefully a sign that stimulus compression is a useful and accurate description of parahippocampal region function (see also Gluck, Myers, \& Goebel, 1994).

Nestor Schmajuk and colleagues have also presented an evolving series of computational models of corticohippocampal interaction in conditioning (Schmajuk \& Blair, 1993; Schmajuk \& Buhusi, 1997; Schmajuk \& DiCarlo, 1991) that make predictions similar to Myers et al.'s (1995) model, but through different learning mechanisms (i.e., error correction and configural learning). The Schmajuk-DiCarlo hippocampal-lesioned model does not exhibit LI, as has been shown in hippocampal-regionlesioned rabbits (Solomon \& Moore, 1975) and in Gluck and Myers's (1993) model.

Later versions of the Schmajuk-DiCarlo model (Schmajuk \& Blair, 1993; Schmajuk \& Buhusi, 1997) differentiated the functions of the hippocampus proper and the entorhinal cortex. Like Myers et al.'s (1995) model, this later model accounts for such effects as intact LI following a selective hippocampallesion (Honey \& Good, 1993; Shohamy et al., 2000). The Schmajuk-DiCarlo model makes no direct predictions concerning LIRR, but we assume that they would predict the same lesion results for this task as they did for LI.

Overall, the Schmajuk-DiCarlo model makes predictions that are consistent with Myers et al.'s (1995) model, but through a different mechanism. Therefore, more work needs to be done to delineate the actual mechanisms operating in the entorhinal cortex during classical conditioning preexposure tasks such as LI and LIRR. This work should be able to determine whether the entorhinal mechanisms of compression (Gluck \& Myers, 1993), fusion (Bunsey \& Eichenbaum, 1993), or configural learning (Schmajuk \& DiCarlo, 1991) are complementary or separable mechanisms.

\section{Conclusions}

Overall, our findings for the work presented here are that nonselective cortical-hippocampal region and selective entorhinal lesions, but not sham or selective hippocampal lesions, disrupt LIRR. These findings, along with these of other lesion studies and theories discussed, indicate that cortical regions within the hippocampal region, such as the entorhinal cortex, play a role in memory that is independent of the hippocampus proper. Whereas nonselective hippocampal region lesion disruption of LIRR indicated that some structure in this brain region was involved in learning and memory, more recent selective lesion work has determined the roles of the specific structures in the hippocampal region. Initially, experimental and theoretical work focused on the hippocampus proper, but more recently this focus has shifted to include nearby cortical regions, such as the entorhinal cortex. This shift in focus from the hippocampus to its related cortical regions is leading to a better understanding of the precise neurobiological mechanisms for learning and memory. Our present findings of LIRR's being disrupted by cortical hippocampal region and selective entorhinal lesions, but not by sham or selective hippocampal lesions, fits with the emerging theory that cortical areas within the hippocampal region may be responsible for tasks previously hypothesized to be hippocampal dependent. Future work needs to continue this line of research in dissociating hippocampal and entorhinal cortical functions in learning and memory.

\section{REFERENCES}

Allen, M. T., Myers, C. E., Schnirman, G., Chelius, L., Masand, V., \& GLUCK, M. A. (2002). Learned irrelevance is a more robust preexposure effect than latent inhibition in eyeblink conditioning in both rabbit and human. Integrative Physiological \& Behavioral Science, $\mathbf{3 7}$, 85-102.

BAKER, A. (1976). Learned irrelevance and learned helplessness: Rats learn that stimuli, reinforcers and responses are uncorrelated. Journal of Experimental Psychology: Animal Behavior Processes, 2, 130-141.

Baker, A., \& Mackintosh, N. (1979). Preexposure to the CS alone, US alone, or CS and US uncorrelated: Latent inhibition, blocking by context or learned irrelevance? Learning \& Motivation, 10, 278-294.

Bennett, C., Maldonado, A., \& Mackintosh, N. (1995). Learned irrelevance is not the sum of exposure to CS and US. Quarterly Journal of Experimental Psychology, 48B, 117-128.

Berger, T. W., \& ORR, W. B. (1983). Hippocampectomy selectively disrupts discrimination reversal conditioning of the rabbit nictitating membrane response. Behavioural Brain Research, 8, 49-68.

BonARDi, C., \& Hall, G. (1996). Learned irrelevance: No more than the sum of CS and US preexposure effects? Journal of Experimental Psychology: Animal Behavior Processes, 22, 183-191.

Buhusi, C. V., Gray, J. A., \& Schmajuk, N. A. (1998). Perplexing effects of hippocampal lesions on latent inhibition: A neural network approach. Behavioral Neuroscience, 112, 316-351.

Bunsey, M., \& Eichenbaum, H. (1993). Critical role for the parahippocampal region for paired-associate learning in rats. Behavioral Neuroscience, 107, 740-747.

Chen, G., \& Steinmetz, J. E. (1998). A general-purpose computer system for behavioral conditioning and neural recording experiments. Behavioral Research Methods, Instruments, \& Computers, 30, 384391.

Cho, Y. H., \& Kesner, R. P. (1996). Involvement of entorhinal cortex or parietal cortex in long-term spatial discrimination memory in rats: Retrograde amnesia. Behavioral Neuroscience, 110, 436-442.

Eichenbaum, H., Otto, T., \& Cohen, N. (1994). Two functional components of the hippocampal memory system. Behavioral \& Brain Sciences, 17, 449-518.

Glasier, M. M., Janis, L. S., Roof, R. L., \& Stein, D. G. (1999). Effects of unilateral entorhinal cortex lesion on retention of water maze performance. Neurobiology of Learning \& Memory, 71, 19-33.

Glasier, M. M., Janis, L. S., \& Stein, D. G. (1997). Persistent short term memory deficits in Hebb-Williams maze performance are shown by rats with unilateral entorhinal cortex lesions. Behavioral Neuroscience, 111, 225-228.

Gluck, M. A., \& Myers, C. E. (1993). Hippocampal mediation of stimulus representation: A computational theory. Hippocampus, 3, 491-516.

Gluck, M. A., \& Myers, C. E. (2001). Getaway to memory. Cambridge, MA: MIT Press.

Gluck, M. A., Myers, C. E., \& Goebel, J. (1994). A computational perspective on dissociating hippocampal and entorhinal function (response to Eichenbaum et al.). Behavioral \& Brain Sciences, 17, 478-479. Gormezano, I., Kehoe, E. J., \& Marshall, B. S. (1983). Twenty years 
of classical conditioning research with the rabbit. Progress in Psychobiology \& Physiological Psychology, 10, 197-275.

Hall, G. (1991). Perceptual and associative learning. Oxford: Oxford University Press, Clarendon Press.

Han, J. S., Gallagher, M., \& Holland, P. C. (1995). Hippocampal lesions disrupt increments but not decrements in stimulus processing. Journal of Neuroscience, 15, 7323-7329.

Holt, W., \& Maren, S. (1999). Muscimol inactivation of the dorsal hippocampus impairs contextual retrieval of fear memory. Journal of Neuroscience, 19, 9054-9062.

Honey, R., \& Good, M. (1993). Selective hippocampal lesions abolish the contextual specificity of latent inhibition and conditioning. Behavioral Neuroscience, 107, 23-33.

JARRARD, L. E. (1986). Selective hippocampal lesions and behavior: Implications for current research and theorizing. In R. L. Isaacson \& K. Pribham (Eds.), The hippocampus (Vol. 4, pp. 93-126). New York: Plenum.

JARRARD, L. E. (1989). On the use of ibotenic acid to lesion selectively different components of the hippocampal formation. Journal of Neuroscience Methods, 29, 251-259.

JARRARD, L. E. (1991). On the use of ibotenic acid to lesion selectively different brain structures. In R. L. Isaacson \& K. Pribram (Eds.), Methods in neuroscience (pp. 58-69). New York: Academic Press.

JARRARD, L. E. (1993). On the role of the hippocampus in learning and memory in the rat. Behavioral \& Neural Biology, 60, 9-26.

JARRARD, L. E., \& DAVIDSON, T. L. (1990). Acquisition of concurrent conditional discriminations in rats with ibotenate lesions of hippocampus and of subiculum. Psychobiology, 18, 68-73.

JARRARD, L. E., \& MeldRUm, B. S. (1993). Selective excitotoxic pathology in the rat hippocampus. Neuropathology \& Applied Neurobiology, 19, 381-389.

Kaye, H., \& Pearce, J. (1987). Hippocampal lesions attenuate latent inhibition and the decline of the orienting response in rats. Quarterly Journal of Experimental Psychology, 39, 107-125.

Lubow, R. (1973). Latent inhibition. Psychological Bulletin, 79, 398 407.

Lubow, R. (1989). Latent inhibition and conditioned attention theory. Cambridge: Cambridge University Press.

Matzel, L., Schact man, T., \& Miller, R. (1988). Learned irrelevance exceeds the sum of CS-preexposure and US preexposure deficits. Journal of Experimental Psychology: Animal Behavior Processes, 14, 311-319.

Myers, C. E., Gluck, M. A., \& Granger, R. (1995). Dissociation of hippocampal and entorhinal function in associative learning: A computational approach. Psychobiology, 23, 116-138.

Otto, T., Wolf, D., \& Walsh, T. J. (1997). Combined lesions of perirhinal and entorhinal cortex impair rats' performance in two versions of the spatially guided radial-arm maze. Neurobiology of Learning \& Memory, 68, 21-31.

Rogers, R. F., Katz, D. B., Gorin, R. E., \& Steinmetz, J. E. (2001). Contextual contributions to the expression of latent inhibition [Compact disk database]. Society for Neuroscience Abstracts, 27.
Schmajuk, N. A., \& Blair, H. T. (1993). Stimulus configuration, place learning and the hippocampus. Behavioural Brain Research, 59, 103 117.

Schmajuk, N. A., \& Buhusi, C. V. (1997). Stimulus configuration, occasion setting, and the hippocampus. Behavioral Neuroscience, 111, 235-258.

Schmajuk, N. A., \& DiCARlo, J. J. (1991). A neural network approach to hippocampal function in classical conditioning. Behavioral Neuroscience, $\mathbf{1 0 5}, 82-110$

Schmajuk, N. A., LAM, Y.-W., \& GRAY, J. A. (1996). Latent inhibition: A neural network approach. Journal of Experimental Psychology: Animal Behavior Processes, 22, 321-349.

Schmajuk, N. A., Lam, Y.-W., \& Christiansen, B. (1994). Latent inhibition of the rat eyeblink response: Effect of hippocampal aspiration lesions. Physiology \& Behavior, 55, 597-601.

Schmajuk, N. A., \& Moore, J. W. (1988). The hippocampus and the classically conditioned nictitating membrane response: A real-time attentional-associative model. Psychobiology, 16, 20-35.

Schnur, P., \& Lubow, R. E. (1976). Latent inhibition:The effects of ITI and CS intensity during preexposure. Learning \& Motivation, 7, 540550.

Shohamy, D., Allen, M. T., \& Gluck, M. A. (2000). Dissociating entorhinal and hippocampal function in latent inhibition. Behavioral Neuroscience, 114, 867-874.

Solomon, P., \& MoORE, J. (1975). Latent inhibition and stimulus generalization of the classically conditioned nictitating membrane response in rabbits (Oryctolagus cuniculus) following dorsal hippocampal ablation. Journal of Comparative \& Physiological Psychology, 89, 1192-1203.

Suzuki, W. A., Zola-Morgan, S., Squire, L. R., \& Amaral, D. G. (1993). Lesions of the perirhinal and parahippocampal cortices in the monkey produce long-lasting memory impairment in the visual and tactual modalities. Journal of Neuroscience, 13, 2430-2451.

Thompson, L. T., Moyer, J. R., Akase, E., \& Disterhoft, J. F. (1994). A system for quantitative analysis of associative learning: Pt. 1. Hardware interfaces with cross-species applications. Journal of Neuroscience Methods, 54, 109-117.

URBAN, I., \& RichaRd, P. (1972). A stereotaxic atlas of the New Zealand rabbit's brain. Springfield, IL: Thomas.

Weiner, I., \& Feldon, J. (1997). The switching model of latent inhibition: An update of neural substrates. Behavioural Brain Research, $\mathbf{8 8}$, 11-25.

YeE, B. K., \& Rawlins, J. N. P. (1998). A comparison between the effects of medial septal lesions and entorhinal cortex lesions on performance of nonspatial working memory tasks and reversal learning. $B e-$ havioural Brain Research, 94, 281-300.

(Manuscript received January 16, 2002; accepted for publication May 21, 2002.) 